\title{
Real-time observation of ultrafast Rabi oscillations between excitons and plasmons in metal nanostructures with J-aggregates
}

\author{
Parinda Vasa ${ }^{1,2 \star}$, Wei Wang1, Robert Pomraenke', Melanie Lammers ${ }^{1}$, Margherita Maiuri', \\ Cristian Manzoni ${ }^{3}$, Giulio Cerullo ${ }^{3}$ and Christoph Lienau ${ }^{1 \star}$
}

Surface plasmon polaritons (SPPs), optical excitations at the interface between a metal and a dielectric, carry significant potential for guiding and manipulating light on the nanoscale $\mathrm{e}^{1-3}$. However, their weak optical nonlinearities hinder active device fabrication, for example, for all-optical switching ${ }^{4-7}$ or information processing ${ }^{8,9}$. Recently, strong optical dipole coupling has been demonstrated between SPPs and nonlinear quantum emitters with normal mode splittings of up to $700 \mathrm{meV}$ (refs 10-15). The predicted ultrafast energy transfer between quantum emitters and SPP fields could be a crucial microscopic mechanism for switching light by light on the nanoscale. Here, we present the first real-time observation of ultrafast Rabi oscillations in a J-aggregate/metal nanostructure, indicating coherent energy transfer between excitonic quantum emitters and SPP fields. We demonstrate coherent manipulation of the coupling energy by controlling the exciton density on a 10 fs timescale, which represents a step towards coherent, all-optical ultrafast plasmonic circuits and devices.

Metallic nanostructures supporting surface plasmon polaritons (SPPs) guide light over ultrashort length and time scales and are finding use in a growing range of applications. Currently, their application is limited primarily by the intrinsically weak optical nonlinearities and short propagation lengths of SPPs ${ }^{16}$. Coupling SPPs to nonlinear optical resonances such as excitons (Xs) in molecular or semiconducting nanostructures can provide the missing nonlinearity and gain. This is a key step towards realizing novel active plasmonic devices such as all-optical switches ${ }^{4,6,7}$, singlephoton transistors ${ }^{5}$, and nanolasers ${ }^{17-19}$, combining the operational bandwidth of photonics with the size scalability of electronics. In appropriately designed nanostructures ${ }^{10-12,15}$, the coupling strength between the optical dipole moment of the exciton and the vacuum SPP field greatly exceeds their individual linewidth, resulting in the formation of hybrid X-SPP modes with energy splittings approaching the exciton resonance energy ${ }^{13}$. These hybrid excitations combine the favourable light-localization properties of SPPs and the enhanced nonlinearities of active quantum emitters. From a dynamical perspective, the strong X-SPP coupling results in a hitherto unexplored periodic and coherent energy exchange between both systems. Here, we study these Rabi oscillations between excitons and SPPs in a J-aggregate/metal hybrid nanostructure in real time. We find that the period of these oscillations (and thus the optical nonlinearity of this hybrid nanostructure) is parametrically modulated on an ultrafast $10 \mathrm{fs}$ timescale by the exciton population, thus providing a new mechanism for ultrafast all-optical switching in active plasmonic devices.
We studied the J-aggregate/metal hybrid nanostructure shown in Fig. 1a, which comprises a J-aggregated cyanine dye embedded in a 50 -nm-thick polymer film, and spin-coated onto periodic nanoslit arrays in a gold film, with period $a_{0}$ ranging from 400 to $460 \mathrm{~nm}$. In such hybrid structures, J-aggregate excitons are strongly coupled via their optical transition dipole moments $\boldsymbol{\mu}_{\mathrm{X}}$ to SPP fields localized in and near the slits. The large oscillator strength of the molecular J-aggregates and the local field enhancement results in particularly large coupling energies ${ }^{7,10,11,13}$. This makes such structures emerging prototypes for investigating the dynamics of the $\mathrm{X}$-SPP hybrid modes ${ }^{4,7,20,21}$. For a single exciton, the strength of this coupling $\hbar \Omega_{\mathrm{R}}=\boldsymbol{\mu}_{\mathrm{X}} \cdot \mathbf{E}_{\mathrm{V}}(\mathbf{r})$ is given by the product of the dipole moment and the amplitude of the SPP vacuum field, $\mathbf{E}_{\mathrm{V}}(\mathbf{r})$ at the exciton position $\mathbf{r}$ (refs 22-24). In our structures, a large number of excitons $\left(N_{X}\right.$; see Supplementary Section S1) are coupled to a quasi-continuum of SPP modes, so we are in the 'classical' limit of a normal mode splitting between excitons and $\mathrm{SPPs}^{25,26}$. In this limit, the linear optical properties of the hybrid system are essentially understood as those of two dissipative oscillators $(\mathrm{X}=i, \mathrm{SPP}=j)$ with complex frequencies $\tilde{\omega}_{i, j(\theta)}=\omega_{i, j(\theta)}-i \gamma_{i, j(\theta)}$, coupled by a nondissipative interaction with strength $\hbar \Omega_{\mathrm{R}}=\sqrt{ }\left(N_{\mathrm{X}}\right) \boldsymbol{\mu}_{\mathrm{X}} \cdot \mathbf{E}_{\mathrm{V}}$. Here $\omega_{i, j(\theta)}$ are the resonance angular frequencies, $\gamma_{i, j}(\theta)$ represents the sum of radiative and nonradiative damping rates caused by coupling to the environment, and $\theta$ is the incidence angle. A strong coupling regime is reached when the normal mode splitting $\Omega_{\mathrm{NMS}} \approx 2\left|\Omega_{\mathrm{R}}\right|$ exceeds all damping rates. This leads to the formation of coupled upper (UP) and lower (LP) X-SPP polariton modes and a characteristic anti-crossing in the dispersion relation (Fig. 1b). Evidence for strong X-SPP coupling is given by recording angle-resolved, $p$-polarized, steady-state reflectivity spectra $R_{0}(\theta, \omega)$, as shown in Fig. 1c. The spectra reveal a significant bending of the X-SPP modes exhibiting an anticrossing with $\hbar \Omega_{\mathrm{NMS}} \approx 110 \mathrm{meV}$. The spectral linewidths indicate lower limits for the dephasing times of the probed ensemble of UP and LP modes of 20 and $40 \mathrm{fs}$, respectively, given by the interplay between radiative damping and exciton dephasing. In addition, a pronounced reflectivity dip is seen at the bare exciton resonance $\omega_{i}$. By comparing its strength to that of the UP and LP resonances at various angles $\theta$, we conclude that only a small fraction $(\sim 0.01)$ of all excitons, namely those situated inside or near the slits, are strongly coupled to the SPP modes. The majority of excitons remain unaffected by the presence of the SPP field. Angle-dependent spectra simulated based on the optical Bloch equation model described in Supplementary Section S1 (Fig. 1d,e) agree well with the experimental spectra and reveal information on the polariton dispersion relations depicted in Fig. 1f.

'Institut für Physik and Center of Interface Science, Carl von Ossietzky Universität, 26129 Oldenburg, Germany, ${ }^{2}$ Department of Physics, Indian Institute of Technology Bombay, 400076 Mumbai, India, ${ }^{3}$ IFN-CNR, Dipartimento di Fisica, Politecnico di Milano, 20133 Milano, Italy. 


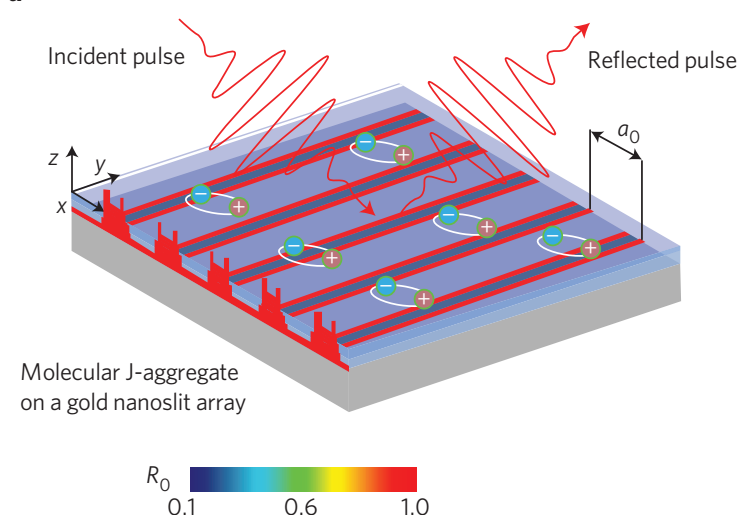

b
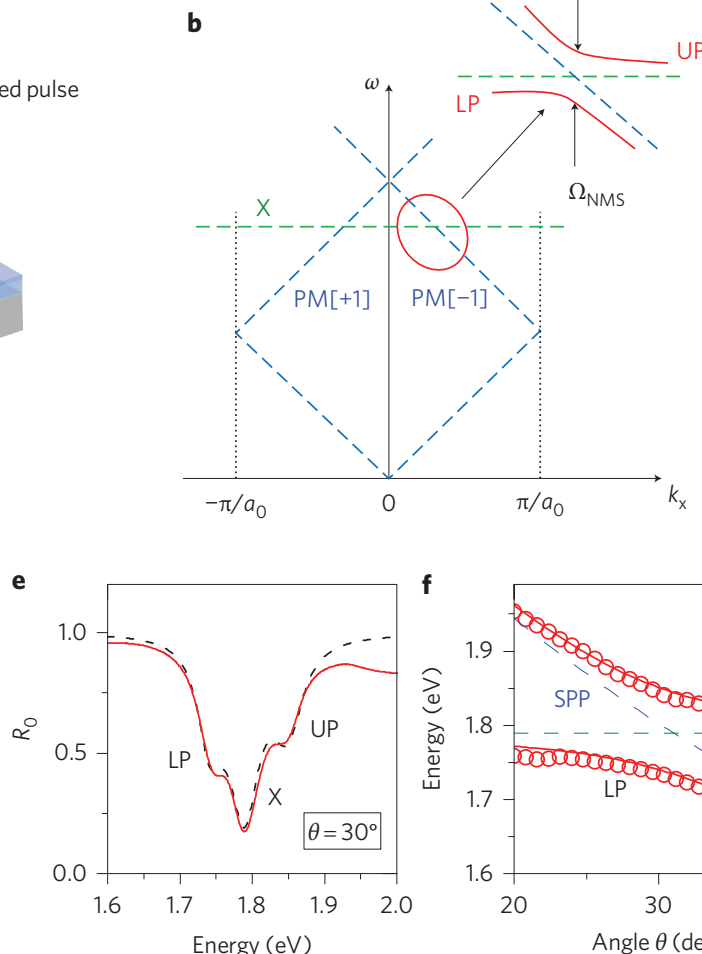

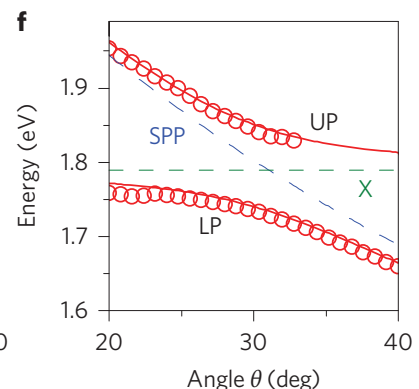

Figure 1 | Strong exciton-SPP coupling in J-aggregate/metal hybrid nanostructures. a, Schematic of coherent ultrafast spectroscopy on a hybrid nanostructure consisting of a 50-nm-thick film of J-aggregate molecules in a polymer matrix coated onto a gold nanoslit array with period $a_{0}$. Strongly localized SPP fields (in red) exist in and near the slits. Incident and reflected laser pulses and J-aggregate excitons are shown schematically. $\mathbf{b}$, Schematic dispersion relations of the exciton $(X)$ and surface plasmon polariton (SPP) resonances at the polymer-metal interface (PM[ \pm 1$]$ ). The strong dipolar $X-S P P$ coupling results in the formation of upper (UP) and lower (LP) exciton SPPs with normal mode splitting $\Omega_{\mathrm{NMS}}$. c, Angle-resolved, $p$-polarized linear reflectivity map $R_{0}(\theta, \omega)$ ( $\theta$, incidence angle), showing a splitting of $\hbar \Omega_{\mathrm{NMS}} \approx 110 \mathrm{meV}$ for a grating with $a_{0}=430 \mathrm{~nm}$. The dispersionless feature at $1.789 \mathrm{eV}$ arises from uncoupled J-aggregate molecules. $\mathbf{d}$, Simulations of $R_{0}(\theta, \omega)$ based on an optical Bloch equation model. e, Experimental (solid line) and simulated (dashed line) reflectivity spectra at $\theta=30^{\circ}$. f, Measured (open circles) and simulated dispersion relations.

In accordance with previous work ${ }^{7,10-12}$, we suggest that a periodic transfer of energy due to the emission and reabsorption of photons is the physical cause for the normal mode splitting. Because of the large value of $\Omega_{\mathrm{NMS}}$, this exchange with a period of $T_{\mathrm{R}} \approx 2 \pi / \Omega_{\mathrm{NMS}}$ is expected to occur on a short timescale of $\sim 30$ fs. To time-resolve these Rabi oscillations and to investigate the coherent polariton dynamics, we performed angle-resolved pump-probe spectroscopy on the hybrid system. Nearly collinearly propagating, $p$-polarized visible pump and probe pulses with sub$15 \mathrm{fs}$ duration and centred at $1.8 \mathrm{eV}$ are weakly focused onto the sample at variable incidence angle $\theta$. The spectral bandwidth covers both polariton branches, impulsively exciting polariton wave packets whose dynamics are probed by monitoring the differential reflectivity spectrum $(\Delta R / R)\left(\omega_{\mathrm{pr}}, \tau\right)$ as a function of probe frequency $\omega_{\mathrm{pr}}$ and time delay $\tau$ between pump and probe pulses.

The time evolution of $\Delta R / R$ is shown in Fig. 2a for a hybrid nanostructure with $a_{0}=430 \mathrm{~nm}$ and $\theta=39^{\circ}$, and excited with a fluence of $30 \mu \mathrm{J} \mathrm{cm}^{-2}$. Here, the SPP mode is detuned by $\sim 75 \mathrm{meV}$ to the red of the exciton resonance at $1.789 \mathrm{eV}$. The data reveal a strong nonlinear signal near $1.789 \mathrm{eV}$ and a weaker signal at the LP resonance $(1.65 \mathrm{eV})$. The $\Delta R$ signal near the LP resonance also exhibits pronounced temporal oscillations. The signal near $1.789 \mathrm{eV}$ is very similar to the nonlinear response of a bare dye film deposited on a planar gold mirror ${ }^{7,27}$. It shows a dispersive line shape reflecting the bleaching of the exciton resonance $(\Delta R>$ $0)$ and the resulting blueshifted exciton to biexciton (XX) absorption $(\Delta R<0)$. Its dynamics mainly reflect the pump-induced perturbation of the exciton free induction decay $(\tau<0$, probe precedes pump) and the decay of the pump-induced exciton population $(\tau>0)$ with a lifetime of $\sim 1$ ps (ref. 7). The nonlinear spectra are well understood in terms of a cascaded X-XX model ${ }^{7,27}$ with a nonlinearity governed by the pump-induced saturation of the exciton absorption (Supplementary Section S4).

We now discuss the nonlinear response at the LP resonance. Here, we also find a dispersive spectral lineshape (Fig. 2b) with $\Delta R>0$ at lower probe energies. The signal shows temporal oscillations with a period of $T_{\mathrm{R}}=27 \mathrm{fs}$ both at positive $(\tau>0)$ and negative delays, and has maxima at integer multiples of $T_{\mathrm{R}}$ (Fig. 2c). These oscillations persist for about $\pm 50 \mathrm{fs}$, suggesting that the radiative damping of the SPP modes ${ }^{28}$ limits the coherence time. Signal amplitude and oscillation period increase when reducing the detuning between the exciton and SPP (compare the trace at $\theta=31^{\circ}$ showing mainly an oscillation with period $T_{\mathrm{R}}=37 \mathrm{fs}$ ). All these observations indicate that the transient oscillations seen on the LP resonance are a distinct signature of coherent X-SPP Rabi oscillations.

To understand the origin of these oscillations, recall that the linear spectra (Fig. 1) show that the absorption coefficient of the SPP mode exceeds by a factor of $\sim 100$ that of the small fraction of those excitons that are coupled to SPP modes. Hence, the broadband pump pulse launches a coherent polariton wave packet that is initially mainly localized on the SPP side. This initiates coherent population oscillations between both states. Our data do not support the assumption that exciton saturation is the dominant nonlinearity. Exciton saturation would result in an absorptive lineshape of the $\Delta R$ spectrum with a large amplitude whenever the wave packet is localized on the exciton side, that is, at $\tau=(2 n+1) T_{\mathrm{R}} / 2$, $n \in \mathbb{Z}$, whereas we observe a dispersive $\Delta R$ spectrum with maxima at integer multiples of the Rabi period, $\tau=n T_{\mathrm{R}}$. This indicates that a 

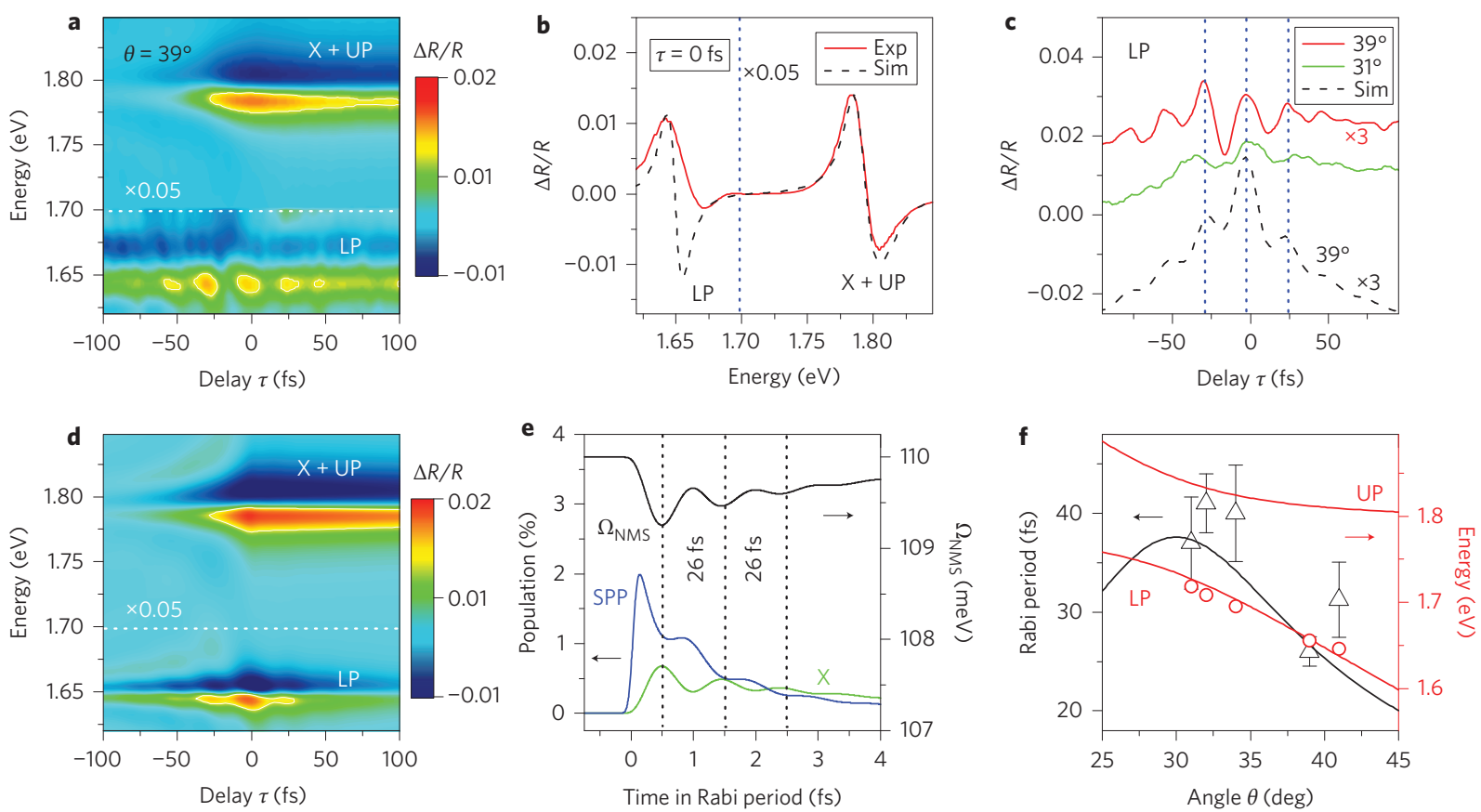

Figure 2 | Coherent dynamics of X-SPP Rabi oscillations. a, Measured differential reflectivity map $(\Delta R / R)\left(\omega_{\mathrm{pr}}, \tau\right)$ for a hybrid structure with $a_{0}=430$ nm, recorded using two nearly collinearly propagating $15 \mathrm{fs}$ pulses with time delay $\tau$ at an incidence angle of $\theta=39^{\circ}$. The pump fluence is set to $30 \mu \mathrm{J} \mathrm{cm}^{-2}$. Clear temporal Rabi oscillations are seen near the LP resonance at $1.65 \mathrm{eV}$. b, Comparison between measured (solid line) and simulated (dashed line) $\Delta R / R$ spectra at $\tau=0$. c, Time evolution of the $\Delta R / R$ signal near the LP resonance measured at two different angles $\theta$, exhibiting pronounced sub-40 fs Rabi oscillations. The shorter oscillation period for $\theta=39^{\circ}$ reflects the increased X-SPP detuning. Simulated dynamics for $\theta=39^{\circ}$ are shown as a dashed line (shifted vertically by -0.025$)$. d,e Simulated $(\Delta R / R)\left(\omega_{\mathrm{pr}}, \tau\right)$ map $(\mathbf{d})$ and pump-induced SPP and exciton population dynamics at $\theta=39^{\circ}$ (e). f, Comparison between observed (open symbols) and calculated (solid lines, error bars taken as standard deviation of Fourier-transformed $\Delta R\left(\omega_{\mathrm{pr}}, \tau\right)$ traces) oscillation periods and LP resonance energies as a function of $\theta$. The simulated dispersion relations are included.

different microscopic mechanism governs the nonlinear response of the hybrid system. Measurements with off-resonant pulsed excitation have shown ${ }^{7}$ that the creation of an exciton population $n_{\mathrm{X}}(t)$ not only bleaches the exciton resonance, but also transiently reduces the normal mode splitting according to $\Omega_{\mathrm{NMS}}(t)=\Omega_{\mathrm{NMS}}^{0} \sqrt{1-2 n_{\mathrm{X}}(t)}$ (Fig. 2e). The material polarization averages over the time-varying normal mode splitting, and this gives rise to a transient blueshift of the lower-frequency LP resonance that is proportional to the time average $\left\langle\Omega_{\mathrm{NMS}}(t)\right\rangle$, resulting in a dispersive LP nonlinearity (Fig. 2b). The shift-and thus the amplitude of $\Delta R$-is large whenever pump and probe pulses create in-phase wave packets at $\tau=n T_{\mathrm{R}}$, that is, when the two wave packets are preferentially localized in the same quantum system. In contrast, it is reduced when out-of-phase wave packets are launched. Hence, a strong pump-induced change in polariton dynamics and thus a large optical nonlinearity is seen at integer multiples of the Rabi period. This dynamical picture is strongly supported by optical Bloch equation simulations (Fig. 2d), reproducing both the dispersive $\Delta R$ lineshape (Fig. $2 \mathrm{~b}$ ) and the oscillatory LP dynamics (Fig. 2c). For this incidence angle, the LP oscillator strength is much larger than that of the UP resonance (Fig. 1), so Rabi oscillations are predominantly seen on the LP branch. When reducing the detuning by changing the incidence angle to $\theta=31^{\circ}$, the Rabi period and the magnitude of the nonlinearity increase in good agreement with our model. The deduced exciton and SPP population, as well as the $\Omega_{\mathrm{NMS}}$ dynamics, are depicted in Fig. 2e. Experiments performed at several values of the detuning are consistent with the scenario described above and provide independent measurements of the Rabi period, with an accuracy limited by the rather short dephasing times, and the LP dispersion relation (Fig. 2f).

These results suggest that the dominant mechanism governing the polariton nonlinearity is a transient reduction in Rabi splitting due to exciton state filling. In Fig. 2a, the decrease in Rabi splitting was rather small (Fig. 2e) and probed by its effect on the lineshape and amplitude of the $\Delta R$ spectrum. To enhance the effect, we increased the pump fluence to $60 \mu \mathrm{J} \mathrm{cm}^{-2}$ and studied the response of the nanostructure in the limit of small detuning $\left(a_{0}=412 \mathrm{~nm}, \theta=33^{\circ}\right.$; Fig. $\left.3 \mathrm{a}\right)$. We observe much stronger dispersive LP nonlinearities, again showing clear signatures of Rabi oscillations (Fig. 3b, blue line). Importantly, the Rabi period decreases progressively with increasing delay. Under these excitation conditions the nonlinear response becomes more complex. The absorption coefficient of the UP branch increases (Fig. 1) and we also see out-of-phase Rabi oscillations on the UP branch, spectrally overlapping with the response of the uncoupled Jaggregate excitons. Measurements on gratings with different periods confirm the assignment of UP and LP resonances (Supplementary Section S6). The observed nonlinear response is again reasonably well explained in terms of our Bloch equation model (Fig. 3c-e). The impulsive excitation launches a coherent SPP wave packet, initiating a population transfer to the exciton system. Consequently, the exciton population reaches a maximum after half a Rabi period and this in turn transiently decreases the normal mode splitting. To approximate the experimentally seen dynamics (Fig. 3b), a rather substantial decrease in normal mode splitting by more than $15 \%$ during the first oscillation cycle (Fig. 3f) should be assumed. The rapid radiative damping of the polariton population progressively reduces the exciton state filling and restores the shorter Rabi period seen in the absence of the pump-induced exciton population (Fig. 3b,f). The amplitude and frequency of this modulation can be controlled by varying the X-SPP mixing ratio and/or pump fluence. The outof-phase oscillations observed on the UP branch reflect the interference between the phase-shifted fields emitted by the hybrid mode and the uncoupled J-aggregate excitons.

In summary, our results present the first real-time observation of coherent X-SPP Rabi oscillations in hybrid nanostructures. They 

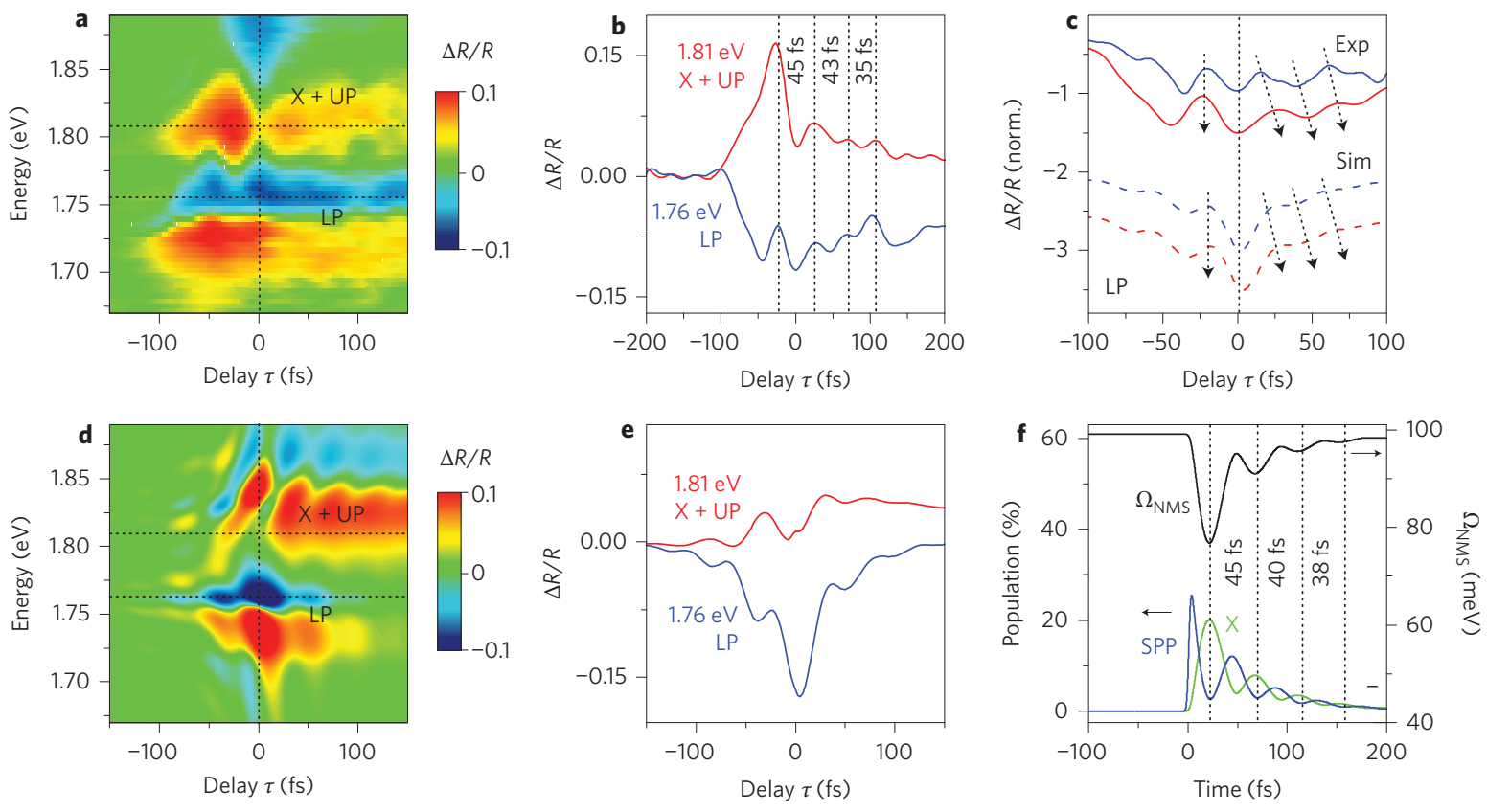

Figure 3 | Transient manipulation of the Rabi energy. $\mathbf{a},(\Delta R / R)\left(\omega_{\mathrm{pr}}, \tau\right)$ spectra at $\theta=33^{\circ}$ for a J-aggregate/metal nanostructure with $a_{0}=412 \mathrm{~nm}$, close to the X-SPP crossing, recorded using sub-15 fs pump pulses with a fluence of $60 \mu \mathrm{J} \mathrm{cm}{ }^{-2}$. The $\Delta R / R$ traces show coherent oscillations with a delay-dependent period. $\mathbf{b}$, Time evolution of $\Delta R / R$ at the LP resonance ( $1.76 \mathrm{eV}$, blue line) and near the overlapping UP and bare J-aggregate resonances at $1.81 \mathrm{eV}$ (red line). c, LP dynamics at low $\left(30 \mu \mathrm{J} \mathrm{cm}^{-2}\right.$, blue) and high $\left(60 \mu \mathrm{J} \mathrm{cm}^{-2}\right.$, red) pump fluence showing an increased and delay-dependent Rabi period at high fluence together with Bloch equation simulations (dashed lines). The traces are shifted vertically for clarity. $\mathbf{d}, \mathbf{e}$, Simulated $\Delta R / R$ spectra (d) and cross-sections (e) at the UP and LP resonances. $\mathbf{f}$, Dynamics of the pump-induced SPP (blue line) and exciton (green line) population together with $\Omega_{\mathrm{NMS}}(t)$ (black line), indicating that a transient modulation of $\Omega_{\mathrm{NMS}}$ is the microscopic origin of the optical nonlinearity of the J-aggregate/metal nanostructure.

show that the optical dipole coupling between excitons and SPPs drastically alters the optical response of the individual resonances. Transient oscillations in exciton density induced by coherent $\mathrm{X}-\mathrm{SPP}$ population transfer give rise to a periodic modulation of the normal mode splitting and thus optical nonlinearity on a $10 \mathrm{fs}$ timescale. This may be seen as a new approach to control the SPP response of metallic nanostructures by coupling SPPs to active quantum systems and providing an ultrafast switching functionality to their otherwise linear response. We believe that this is a promising route towards active all-optical nanophotonic circuits and devices.

\section{Methods}

Sample fabrication. Nanoslit arrays with periods in the range $400-460 \mathrm{~nm}$, depth of $30 \mathrm{~nm}$, slit width of $45 \mathrm{~nm}$ and area of $150 \mu \mathrm{m} \times 150 \mu \mathrm{m}$ were fabricated on an optically thick gold film by focused ion beam milling (FEI Helios NanoLab 600i). Such arrays give rise to significant field confinement inside the slits and near the slit edges (Fig. 1a). Simulations of the electric field distribution are presented in Supplementary Section S2. The cyanine dye 2,2' -dimethyl-8-phenyl-5,6,5', $6^{\prime}$ dibenzothiacarbocyanine chloride (Hayashibara Bio-chemicals Laboratories) was dissolved in a solution containing polyvinyl alcohol (PVA, 80\% hydrolysed, Sigma Aldrich, $26 \mathrm{mg})$, water $(1 \mathrm{ml})$ and methanol $(4 \mathrm{ml})$ and was spin-coated at 8,000 r.p.m. onto the nanoslit arrays to deposit a 50 -nm-thick film. The concentration of the dye in dry PVA was typically $0.5 \mathrm{~mol} \mathrm{dm}^{-3}$, resulting in an absorption coefficient ${ }^{7,11}$ of $\sim 2 \times 10^{5} \mathrm{~cm}^{-1}$. The slit-array period $a_{0}$ of $400-460 \mathrm{~nm}$ was chosen so that the first-order polymer-metal plasmon (PM[-1]) mode was in resonance with the J-aggregate exciton at $\sim 1.789 \mathrm{eV}$. Although the coupling energy is somewhat reduced ${ }^{7,11,15}$, we used thinner dye films and narrow, shallow slit arrays to minimize radiative SPP damping ${ }^{28}$ and to increase the polariton lifetime. The geometric parameters were optimized such that the array acted as a plasmonic resonator, allowing optical confinement of the far-field light predominantly within the nanoslits. The optical properties of the optically thick nanoslit array were studied in reflection geometry.

Experimental. All experiments were performed using an angle-resolved reflectivity setup with an angular resolution of $0.2^{\circ}$. The $p$-polarized linear reflectivity spectra were recorded using a coherent white-light source (Fianium SC-450-4), whereas transient $\Delta R / R$ measurements were performed in a femtosecond pump-probe spectrometer (Supplementary Section S3). The setup began with a regeneratively amplified mode-locked Ti:sapphire laser system (Quantronix Integra C) delivering pulses with a duration of $150 \mathrm{fs}$ and energy of $500 \mu \mathrm{J}$ at a repetition rate of $1 \mathrm{kHz}$ and with $1.6 \mathrm{eV}$ photon energy. The system drove a non-collinear optical parametric amplifier generating broadband near-infrared pulses at $1.8 \mathrm{eV}$ and with a spectrum extending from 1.65 to $1.9 \mathrm{eV}$ (ref. 29). These pulses were compressed to sub-15 fs duration by multiple bounces on chirped mirrors (Venteon) and were split into a pair of $p$-polarized pump and probe pulses. The pulses were shifted slightly vertically, along the slit axis, and were nearly collinearly focused onto the sample to a beam diameter of $\sim 100 \mu \mathrm{m}$ at an incidence angle of $\sim 30^{\circ}$ with respect to the sample normal. The data in Fig. 2 and the low-power curve in Fig. $3 c$ were obtained with pump and probe pulse energies of 2.5 and $0.5 \mathrm{~nJ}$, corresponding to fluences of 30 and $6 \mu \mathrm{J} \mathrm{cm}^{-2}$, whereas the measurements in Fig. 3a were performed at fluences of $60 \mu \mathrm{J} \mathrm{cm}{ }^{-2}$ and $30 \mu \mathrm{J} \mathrm{cm}^{-2}$, respectively. One of the pump beam mirrors was vibrated to suppress interference artefacts in the $\Delta R / R$ spectra close to zero delay. All experiments were performed at room temperature under vacuum to minimize Jaggregate photobleaching.

Theoretical modelling. As described in Supplementary Section S1, the hybrid $\mathrm{X}-\mathrm{SPP}$ polariton modes are given as a coherent superposition of exciton and SPP wavefunctions, $|U P, L P\rangle=((\Delta \pm A)|i\rangle+2 D|j\rangle) / B_{ \pm}$. Their characteristic eigenfrequencies are given as $\tilde{\omega}_{\mathrm{UP}, \mathrm{LP}}=(1 / 2)\left(\tilde{\omega}_{i}+\tilde{\omega}_{j(\theta)} \pm A\right)$, where $A=\sqrt{\Delta^{2}+4 C D}, \Delta=\tilde{\omega}_{i}-\tilde{\omega}_{j(\theta)}, C=\Omega_{R}-i \gamma_{i j}$ and $D=\Omega_{R}^{*}-i \gamma_{i j}$. The normalization constant $B_{ \pm}=\sqrt{|\Delta \pm A|^{2}+4|D|^{2}}$, and $\gamma_{i j}$ is a rate for incoherent photon exchange giving rise to collective spontaneous emission of the coupled system $^{30}$. The measured linear and differential reflectivity spectra are simulated by solving optical Bloch equations for the system of interacting excitons and SPPs using the density matrix formalism. In the simulations, the exciton system is modelled as an effective three-level system (ground state, X, XX), whereas the SPP mode is described as a photon-like harmonic oscillator. The simulations include dipole coupling between exciton and $\mathrm{SPP}^{30}$ and a semiclassical coupling of both systems to an external laser field. Radiative damping and pure dephasing of excitons is implemented phenomenologically by following the Lindblad formalism ${ }^{30}$. In our samples, the SPP fields are mainly confined inside or near the slits, so the Rabi splitting is strongly reduced for those excitons located away from the slits. Contribution from such weakly interacting 'uncoupled' dye molecules has also been taken into account. A detailed description of the density matrix calculations together with estimates of the coupling parameters are given in Supplementary Section S1.

Received 19 July 2012; accepted 4 December 2012; published online 20 January 2013 


\section{References}

1. Maier, S. A. et al. Local detection of electromagnetic energy transport below the diffraction limit in metal nanoparticle plasmon waveguides. Nature Mater. 2, 229-232 (2003).

2. Bozhevolnyi, S. I., Volkov, V. S., Devaux, E., Laluet, J. Y. \& Ebbesen, T. W. Channel plasmon subwavelength waveguide components including interferometers and ring resonators. Nature 440, 508-511 (2006).

3. Lal, S., Link, S. \& Halas, N. J. Nano-optics from sensing to waveguiding. Nature Photon. 1, 641-648 (2007).

4. Dintinger, J., Robel, I., Kamat, P., Genet, C. \& Ebbesen, T. Terahertz all-optical molecule-plasmon modulation. Adv. Mater. 18, 1645-1648 (2006).

5. Chang, D. E., Sorensen, A. S., Demler, E. A. \& Lukin, M. D. A single-photon transistor using nanoscale surface plasmons. Nature Phys. 3, 807-812 (2007).

6. MacDonald, K. F., Samson, Z. L., Stockman, M. I. \& Zheludev, N. I. Ultrafast active plasmonics. Nature Photon. 3, 55-58 (2009).

7. Vasa, P. et al. Ultrafast manipulation of strong coupling in metal-molecular aggregate hybrid nanostructures. ACS Nano 4, 7559-7565 (2010).

8. Engheta, N. Circuits with light at nanoscales: optical nanocircuits inspired by metamaterials. Science 317, 1698-1702 (2007).

9. Gonzalez-Tudela, A. et al. Entanglement of two qubits mediated by onedimensional plasmonic waveguides. Phys. Rev. Lett. 106, 020501 (2011).

10. Bellessa, J., Bonnand, C., Plenet, J. C. \& Mugnier, J. Strong coupling between surface plasmons and excitons in an organic semiconductor. Phys. Rev. Lett. 93, 036404 (2004).

11. Dintinger, J., Klein, S., Bustos, F., Barnes, W. L. \& Ebbesen, T. W. Strong coupling between surface plasmon-polaritons and organic molecules in subwavelength hole arrays. Phys. Rev. B 71, 035424 (2005).

12. Hakala, T. K. et al. Vacuum Rabi splitting and strong-coupling dynamics for surface-plasmon polaritons and rhodamine 6G molecules. Phys. Rev. Lett. 103, 053602 (2009).

13. Schwartz, T., Hutchison, J. A., Genet, C. \& Ebbesen, T. W. Reversible switching of ultrastrong light-molecule coupling. Phys. Rev. Lett. 106, 196405 (2011).

14. Guebrou, S. A. et al. Coherent emission from a disordered organic semiconductor induced by strong coupling with surface plasmons. Phys. Rev. Lett. 108, 066401 (2012).

15. Fofang, N. T. et al. Plexcitonic nanoparticles: plasmon-exciton coupling in nanoshell-J-aggregate complexes. Nano Lett. 8, 3481-3487 (2008).

16. Stockman, M. I. Nanoplasmonics: past, present, and glimpse into future. Opt. Express 19, 22029-22106 (2011).

17. Bergman, D. J. \& Stockman, M. I. Surface plasmon amplification by stimulated emission of radiation: quantum generation of coherent surface plasmons in nanosystems. Phys. Rev. Lett. 90, 027402 (2003).

18. Noginov, M. A. et al. Demonstration of a spaser-based nanolaser. Nature 460 , 1110-1112 (2009).

19. Oulton, R. F. et al. Plasmon lasers at deep subwavelength scale. Nature 461, 629-632 (2009)

20. Zheludev, N. I., Prosvirnin, S. L., Papasimakis, N. \& Fedotov, V. A. Lasing spaser. Nature Photon. 2, 351-354 (2008)
21. Fofang, N. T., Grady, N. K., Fan, Z., Govorov, A. O. \& Halas, N. J. Plexciton dynamics: exciton-plasmon coupling in a J-aggregate-Au nanoshell complex provides a mechanism for nonlinearity. Nano Lett. 11, 1556-1560 (2011).

22. Thompson, R. J., Rempe, G. \& Kimble, H. J. Observation of normal-mode splitting for an atom in an optical cavity. Phys. Rev. Lett. 68, 1132-1135 (1992).

23. Reithmaier, J. P. et al. Strong coupling in a single quantum dot-semiconductor microcavity system. Nature 432, 197-200 (2004).

24. Yoshie, T. et al. Vacuum Rabi splitting with a single quantum dot in a photonic crystal nanocavity. Nature 432, 200-203 (2004).

25. Weisbuch, C., Nishioka, M., Ishikawa, A. \& Arakawa, Y. Observation of the coupled exciton-photon mode splitting in a semiconductor quantum microcavity. Phys. Rev. Lett. 69, 3314-3317 (1992).

26. Khitrova, G., Gibbs, H. M., Jahnke, F., Kira, M. \& Koch, S. W. Nonlinear optics of normal-mode-coupling semiconductor microcavities. Rev. Mod. Phys. 71, 1591-1639 (1999).

27. Fidder, H., Knoester, J. \& Wiersma, D. A. Observation of the one-exciton to two-exciton transition in a J aggregate. J. Chem. Phys. 98, 6564-6566 (1993).

28. Kim, D. S. et al. Microscopic origin of surface-plasmon radiation in plasmonic band-gap nanostructures. Phys. Rev. Lett. 91, 143901 (2003).

29. Manzoni, C., Polli, D. \& Cerullo, G. Two-color pump-probe system broadly tunable over the visible and the near infrared with sub-30 fs temporal resolution. Rev. Sci. Instrum. 77, 023103 (2006).

30. Akram, U., Ficek, Z. \& Swain, S. Decoherence and coherent population transfer between two coupled systems. Phys. Rev. A 62, 013413 (2000).

\section{Acknowledgements}

The authors thank the Deutsche Forschungsgemeinschaft (SPP 1391 and DFG-NSF Materials World Network), Fondazione Cariplo ('Engineering of optical nonlinearities in plasmonic metamaterials'), European Community (FP-7 INFRASTRUCTURES-2008-1, 'Laserlab Europe II', contract no. 228334 and FP-7 NMP 'Cronos') and the Korea

Foundation for International Cooperation of Science and Technology (Global Research Laboratory project, K20815000003) for financial support. The authors also thank E. Sommer for preparing some of the figures.

\section{Author contributions}

P.V., R.P., W.W. and C.L. designed and fabricated the hybrid nanostructures. All authors participated in conducting the experiments. R.P., P.V., W.W. and C.L. contributed to the theoretical modelling. All authors discussed the results and implications at all stages. P.V., R.P., G.C. and C.L. wrote the paper.

\section{Additional information}

Supplementary information is available in the online version of the paper. Reprints and permission information is available online at http://www.nature.com/reprints. Correspondence and requests for materials should be addressed to P.V. and C.L.

\section{Competing financial interests}

The authors declare no competing financial interests. 